\title{
An Unsupervised and Non-parametric Bayesian Image Segmentation
}

\author{
M. Zribi and F. Ghorbel \\ Groupe Recherche Images et Forme \\ Institut National des Télécommunications, ENIC - INT \\ cité scientifique, rue G. Marconi, 59650 \\ Villeneuve d'Ascq FRANCE.
}

\begin{abstract}
We propose here an unsupervised Bayesian image segmentation based on a non-parametric Expectation-Maximisation (EM) algorithm. The non-parametric aspect comes from the use of the orthogonal probability density function (pdf) estimation, which is reduced to the estimation of the first Fourier coefficients (FC's) of the pdf with respect to a given orthogonal basis. So, the mixture identification step based on the maximisation of the likelihood can be realised without hypothesis on the distribution of the conditional pdf. This means that we do not need some assumption for the gray level image pixels distribution. The generalisation to the multivariate case can be obtained by considering the multidimensional orthogonal function basis. In this paper, we intend to give some simulation results for the determination of the smoothing parameter. This algorithm is applied to a contextual image segmentation. Such method conjugated with Bootstrap Sampling allows as the exploration of a large neighbourhood context.
\end{abstract}

\section{Introduction}

It is well known that the Bayesian classification rule is the best one in the mean of the a posterior probability of miss-classification. However, the application of this rule in an unsupervised context needs some parametric hypothesis for the conditional probability distributions of each class. Using the orthogonal pdf estimator, we intend to introduce an original non-parametric and unsupervised Bayesian classifier of which we will prove the robustness in the image segmentation. The unsupervised Bayesian classification consists of the two following important steps : The first one is the mixture identification. It can be achieved by the Expectation-Maximisation (EM) algorithm which was proposed in 1977 [1] for parametric unsupervised Bayesian classification. It is an iterative procedure which assumes that the mixture pdf of data is a linear combination of a finite number of Gaussian distributions where the coefficients of this combination are the a priori probability of each class. The second step consists of the application of the Bayesian classification rule. Using the orthogonal pdf estimator [4], a non-parametric segmentation can be realised. Effectively, this estimator is reduced to the estimation of a finite number of parameters. These features are the FC's of the conditional pdf of each class. During 
iterations, the number of FC's to estimate changes since it depends on the seize of the sample belonging to each class.

In this article, we propose to apply this algorithm to the segmentation of real images. We use the Bootstrap sampling $[2,3]$ in order to reduce the dependence between the pixel sample and the computing time without destroying the quality of the segmented image. The reduction of computing time allows us the exploration of the high dimensional contextual segmentation of images.

\section{The classical EM algorithm}

The classical EM algorithm assumes that the observed image is a realisation of a mixture distributions, so that its pdf can be written as:

$$
f(x)=\sum_{j=1}^{K} \pi_{j} f\left(x / \theta_{j}\right) \text {, with } 0 \leq \pi_{j} \leq 1 \text { and } \sum_{j=1}^{K} \pi_{j}=1
$$

where $f\left(x / \theta_{j}\right)$ is the conditional pdf and $\pi_{j}$ is the probability a priori of class $j$. This algorithm is iterative and has the three following steps. Gaussian hypothesis are generally taken (i.e: $\theta_{j}=\left(\mu_{j}, \sigma_{j}^{2}\right)$ where $\mu_{j}$ represents the mean and $\sigma_{j}^{2}$ is the variance of the class $j$ ).

- Initialization step: The number of classes $\mathrm{K}$ is assumed to be known. An initial solution of the parameters of the mixture are extracted from the histogram.

- Expectation step: This consists on the estimation of the a posterior probability $\hat{\pi}_{j}^{n}\left(x_{i}\right)$ for the pixel $x_{i}$ belonging to the class $j$ at the $n$-th iteration:

$$
\hat{\pi}_{j}^{n}\left(x_{i}\right)=\frac{\hat{\pi}_{j}^{n} f\left(x_{i} / \hat{\theta}_{j}^{n}\right)}{\sum_{i=1}^{K} \hat{\pi}_{1}^{n} f\left(x_{i} / \hat{\theta}_{1}^{n}\right)}
$$

-Maximization step: The parameters needed for the next step are constructed, in the following way:

$$
\hat{\pi}_{j}^{n+1}=\frac{1}{N} \sum_{i=1}^{N} \hat{\pi}_{j}^{n}\left(x_{i}\right), \hat{\mu}_{j}^{n+1}=\frac{\sum_{i=1}^{N} x_{i} \hat{\pi}_{j}^{n}\left(x_{i}\right)}{\sum_{i=1}^{N} \hat{\pi}_{j}^{n}\left(x_{i}\right)} \text { and }\left[\hat{\sigma}_{j}^{2}\right]^{n+1}=\frac{\sum_{i=1}^{N} \hat{\pi}_{j}^{n}\left(x_{i}\right)\left(x_{i}-\hat{\mu}_{j}^{n+1}\right)\left(x_{i}-\hat{\mu}_{j}^{n+1}\right)^{T}}{\sum_{i=1}^{N} \hat{\pi}_{j}^{n}\left(x_{i}\right)}
$$

\section{The proposed non-parametric EM}

\subsection{Estimation based on orthogonal expansions}

The estimation of the pdf based on Fourier analysis methods is suitable for this context. Then an estimator of the pdf based on a independent sample $X_{1}, \ldots \ldots, X_{N}$ with density $f$ is given by: 
$\hat{f}_{k_{s}}(x)=\sum_{m=0}^{K_{N}} \hat{a}_{m, N} e_{m}(x)$ where $\hat{a}_{m, N}=\frac{1}{N} \sum_{i=1}^{N} e_{m}\left(X_{i}\right),\left\{e_{m}(x)\right\}_{m \in \mathbb{N}}$ is an orthonormal complete basis of the Hilbert space $L_{2}([a, b])$ and $K_{N}$ is called truncation point.

\subsection{Description of the non-parametric EM Segmentation}

The kind of the conditional pdfs of a gray level of image pixels do not need to be known with this approach, since we propose to define $\theta_{j}$ of section 2 as:

$$
\theta_{j}=\left(a_{0, j}, \ldots \ldots \ldots, a_{k_{N_{j}}, j}\right) \text {. So we denote by } \hat{f}_{k_{\mathrm{k}_{j}}}(x)=f\left(x / \hat{\theta}_{j}\right)
$$

In the following, we intend to describe the proposed algorithm. It consists of three steps:

a. Initialization step : Using the histogram, the observed sample are arranged in $\mathrm{K}$ groups. So, the parameters of mixture can be initialised as following:

$$
\hat{\pi}_{j}^{0}=\frac{\hat{N}_{j}^{0}}{N}, K_{\hat{N}_{j}^{0}}=\operatorname{int}\left[\left(\hat{N}_{j}^{0}\right)^{1 / s}\right] \text { and } \hat{a}_{m, j}^{0}=\frac{1}{\hat{N}_{j}^{0}} \sum_{l=1}^{\hat{N}_{j}^{0}} e_{m}\left(X_{l}\right)
$$

where int $[x]$ is the biggest integer is inferior to the real number $x$.

b. Expectation step : In this step, we estimate the a posterior probability $\hat{\pi}_{j}^{n}\left(x_{i}\right)$ for the pixel $x_{i}$ belonging to the class $j$ at the $n$-th iteration :

$$
\hat{\pi}_{j}^{n}\left(x_{i}\right)=\frac{\hat{\pi}_{j}^{n} f\left(x_{i} / \hat{\theta}_{j}^{n}\right)}{\sum_{1=1}^{K} \hat{\pi}_{1}^{n} f\left(x_{i} / \hat{\theta}_{j}^{n}\right)}
$$

c. Maximization step : The a posterior probability $\hat{\pi}_{j}^{n}\left(x_{i}\right)$ of each pixel $x_{i}$ is computed. So that, at $(n+1)$-th iteration, we have:

$$
\begin{gathered}
\hat{\pi}_{j}^{n+1}=\frac{1}{N} \sum_{l=1}^{N} \hat{\pi}_{j}^{n}\left(x_{1}\right), K_{\hat{N}_{j}^{n+1}}=\operatorname{int}\left[\left(\hat{N}_{j}^{n+1}\right)^{1 / s}\right] \text { where } \hat{N}_{j}^{n+1}=N \hat{\pi}_{j}^{n+1} \\
\hat{a}_{m, j}^{n+1}=\frac{\sum_{l=1}^{N} e_{m}\left(x_{1}\right) \hat{\pi}_{j}^{n}\left(x_{1}\right)}{\sum_{l=1}^{N} \hat{\pi}_{j}^{n}\left(x_{1}\right)} \text { for } m=0, \ldots \ldots, K_{\hat{N}_{j}^{n+1}}
\end{gathered}
$$

The Bayesian rule: After the mixture identification, the Bayesian rule is applied in order to classify the pixels according their gray level $\mathrm{x}$ :

$$
j(x)=\operatorname{Arg}\left[\max _{1 \leq j \leq k}\left\{\pi_{j} f\left(x / \theta_{j}\right)\right\}\right]
$$

where $j(x)$ represents the label of the class of the pixel $x$. 


\section{Smoothing parameters estimation}

It is well known that the orthogonal estimation method depends on the smoothing parameter which we notice $1 / \mathrm{s}\left(\mathrm{k}_{\mathrm{N}} \approx \mathrm{N}^{1 / 5}\right)$. Convergence theorems are well studied for finding the optimal values of $1 / \mathrm{s}[4,5,6]$. However, in the mixture identification, such problem appears to be more difficult. In order to give an idea on the values of $1 / \mathrm{s}$, we propose here some simulation studies. We randomly generate a Gaussian mixture distribution. So, the proposed pdf estimation of the mixture can be written as:

$$
\hat{\mathrm{f}}_{\mathrm{t} / \mathrm{s}}(\mathrm{x})=\sum_{\mathrm{j}=1}^{\mathrm{x}} \hat{\pi}_{\mathrm{j}} \mathrm{f}\left(\mathrm{x} / \hat{\theta}_{\mathrm{j}}\right) \text { with } \hat{\theta}_{\mathrm{j}}=\left(\hat{\mathrm{a}}_{0, \mathrm{j}}, \ldots \ldots \ldots, \hat{\mathrm{a}}_{\mathrm{K}_{\mathrm{j} j} \mathrm{j}}\right)
$$

Where $\hat{\theta}_{\mathrm{j}}=\left(\hat{\mathrm{a}}_{0, \mathrm{j}}, \ldots \ldots \ldots, \hat{\mathrm{a}}_{\mathrm{K}_{\mathrm{y}}, \mathrm{j}}\right)$ are the estimated FC's of the conditional pdfs and $\mathrm{K}_{\mathrm{Nj}}$ is int $\left[\mathrm{N}_{i}^{1 / s}\right]$ for some values of $s \geq 1$. This asymptotic study is realised in the sense of mean integrated square error (MISE) between the theoretical pdf $f$ and $\hat{f}_{1 / s}$ :

$$
E\left[d_{2}\left(f, \hat{f}_{1 / s}\right)\right]=g(1 / s)
$$

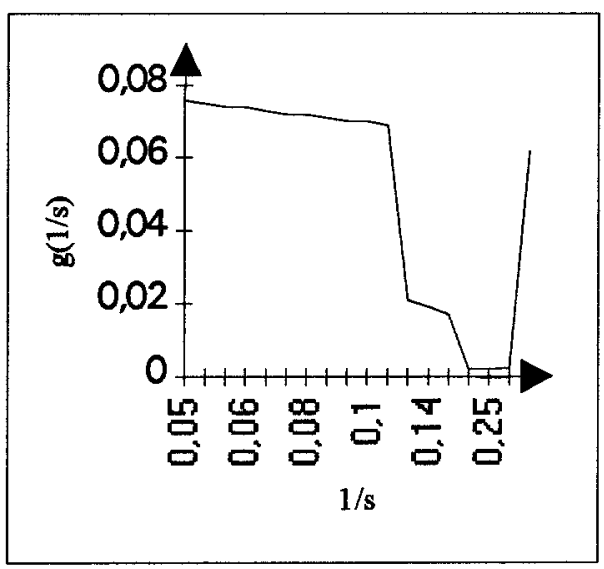

Fig. 0

Fig. 0. represents the MISE according $1 / \mathrm{s}$ for a Gaussian mixture. We can observe that the optimal values of $1 / \mathrm{s}$ are about 0.25 . This result can be shown for kind of mixtures distribution. So, the optimal values of $1 / \mathrm{s}$ are similar to those the classical theoretical results for the non mixture case $[4,5,6]$. 


\section{Non-parametric image segmentation}

The non-parametric and unsupervised Bayesian classifier is applied to Retina image, and traffic signs image. The original images are represented by 256 gray levels. We consider three classes. Trigonometric basis are used for the non-parametric mixture identification step. However, Gaussian mixture is assumed for the classical EM segmentation procedure. It improves the segmentation result with respect the classical EM procedure for the traffic images (see Fig 2, 4,6).

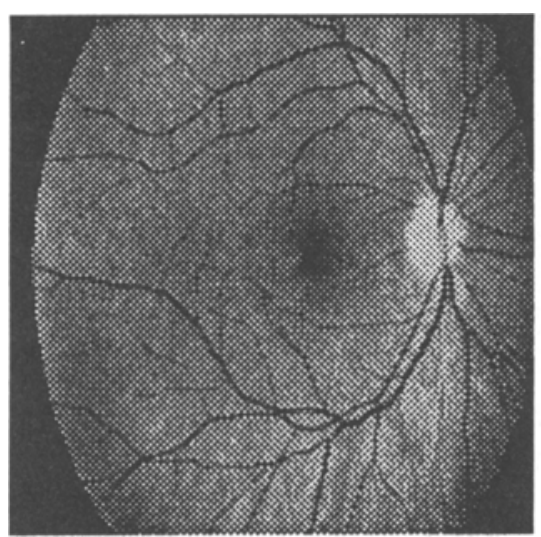

Fig. 1. Original Image

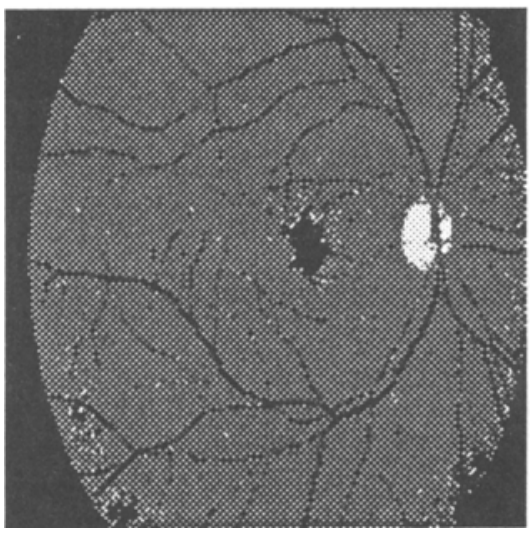

Fig. 3. Segmented Image with Gaussian mixture

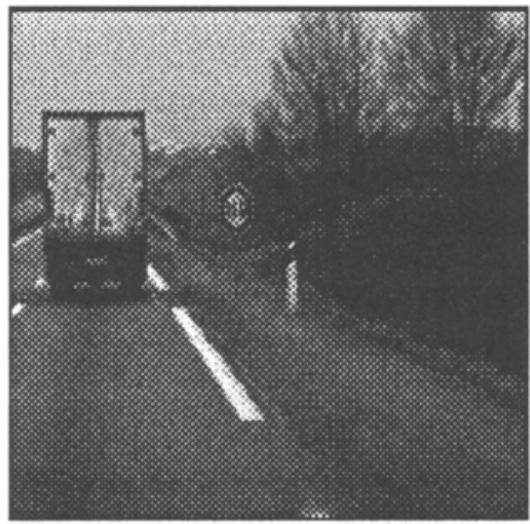

Fig. 2. Original Image

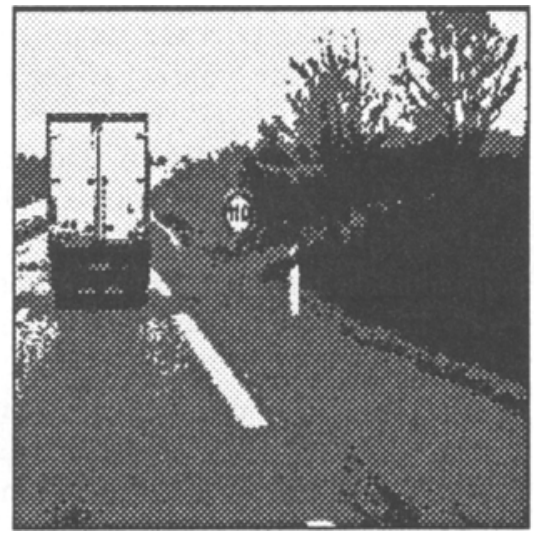

Fig. 4. Segmented Image with Gaussian mixture 


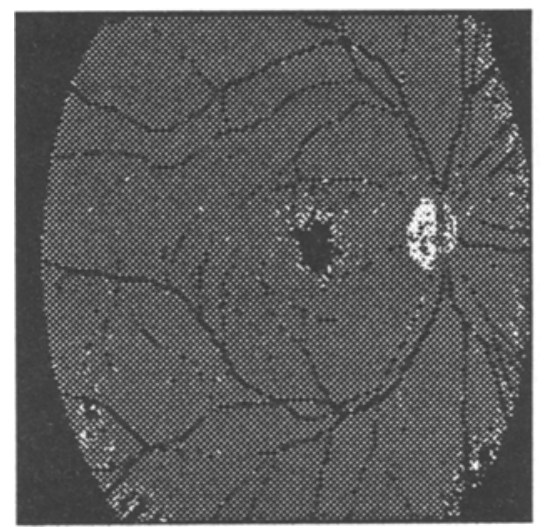

Fig. 5. Segmented Image with non-parametric EM

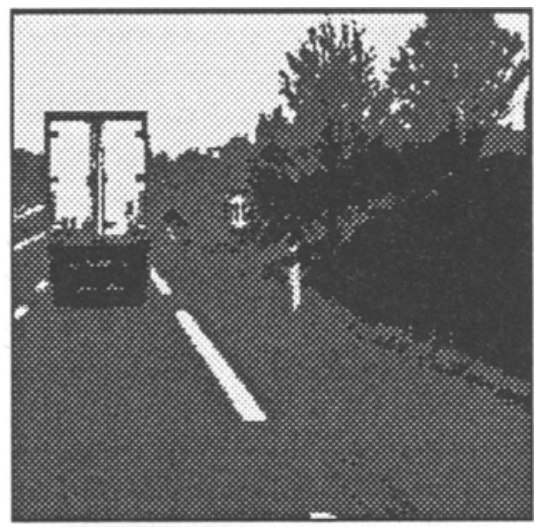

Fig. 6. Segmented Image with non-parametric EM

\section{Perspectives:}

The extension to multivariate case of the classical EM algorithm for non Gaussian multivariate pdf's is not trivial. However, the generalisation of the proposed algorithm to the contextual case can be done easily since the orthogonal function basis in the multivariate case could be obtained by the product of one dimensional orthogonal system functions.

\section{References}

1. A. P. Dempester et al: Maximum Likelihood from Incomplete Data via the E.M Algorithm. Journal of the Royal Statistical Society, Series B, 39:1, 38 (1977).

2. C. Banga et al: Optimal Bootstrap Sampling for fast image Segmentation, Application to Retina Image. IEEE-ICASSP 1993, April 27-30 Minneapolis USA.

3. F. Ghorbel et al: Bootstrap sampling approach in pattern recognition, The special session, the Bootstrap and Its Application. ICASSP April 94 South of Australia.

4. R. Kronmal et al: The estimation of probability and cumulatives by Fourier series methods. J.Amer Statist. Ass, Vol-63, pp 925-952, 1968.

5. P. Hall: Measuring the efficiency of trigonometrique series estimates of density. J.Multivarate Anal, in press, 1981 .

6. P. Hall: Comparison of two orthogonal series methods of estimating a density and its derivatives on a interval. Journal of multivariate analysis, 12, 432$449,1982$. 